ad-hoc basis with several specialist pharmacists reviewing queries on a daily basis. Average call durations were between 5 to 8 minutes with more complex queries requiring in depth data search taking up to 30 minutes. All queries are logged on paper and then reviewed on a monthly basis as they are entered onto a database. Since the introduction of the service, the volume of calls received has increased by more than 50\% with average of 35 per month in 2015 and 54 in 2017. Originally, the service was designed primarily for patients, parents and carers. Due to the increased recognition, the service has now been expanded to a variety of internal and external healthcare professionals, community practitioners and pharmacies, drug companies, commissioning staff, researchers and students. The types of queries range from supply issues, procurement of unlicensed medicines, to adverse effects, administration advice and complex pharmaceutical queries.

Conclusion The service has grown and developed with focus based around improving patient care, medication adherence and minimising medicines related risks. Through providing accurate, up-to-date and evidence based information its appeal has reached a wider audience including healthcare professionals. Combined with an increase in the number of calls and technological advances, a new email service has been rolled out in 2017, as an alternate means to contact the service. Direct comments from users of the service has shown positive feedback and trust.

\section{P044 CAN AN ELECTRONIC PRESCRIBING SYSTEM ENSURE CLEAR ANTICOAGULATION DISCHARGE COMMUNICATION?}

Susie Gage. Bristol Royal Hospital for Children

\subsection{6/archdischild-2019-nppc.53}

Aim The National Patient Safety Agency (NPSA) ${ }^{1}$ identified heparin as a major cause of adverse events associated with adverse incidents, including some fatalities. By ensuring good communication, this should be associated with risk reduction. ${ }^{1}$ The aim of this study was to ensure there is clear anticoagulation communication on discharge, from the paediatric intensive care unit (PICU) electronic prescribing system (Philips), to the paediatric cardiac high dependency unit and paediatric cardiac ward. To investigate whether the heparin regimen complies with the hospital's anticoagulant guidelines and if there is any deviation; that this is clearly documented. To find out if there is an indication documented for the heparin regimen chosen and if there is a clear long term plan documented for the patient, after heparin cessation.

Methods A report was generated for all patients who were prescribed a heparin infusion on PICU, between 1st January 2018 and 30th June 2018, from the Philips system. All discharge summaries from the PICU Philips system were reviewed. Only paediatric cardiac patients were included that had a heparin infusion prescribed on discharge, all other discharge summaries were excluded from the study. Each discharge summary was reviewed in the anticoagulant section; for the heparin regimen chosen, whether it complies with the hospital's anticoagulant guidelines and if there was any deviation whether this was documented. The indication documented of which heparin regimen was chosen and whether a clear long term plan was documented after heparin cessation; for example if the patient is to be transferred onto aspirin, clopidogrel, warfarin or enoxaparin.

Results 82 discharge summaries were reviewed over the 6 month period between 1st January 2018 and 30th June 2018; 16 were excluded as were not paediatric cardiac, leaving 66 paediatric cardiac discharge summaries that were reviewed. 45 out of $66(68 \%)$ complied with the hospital's heparin anticoagulation guidelines. Of the $32 \%$ that deviated from the protocol; only $33 \%$ (7 out of 21 ) had a reason documented. Only $50 \%$ (33) of the summaries reviewed had an indication for anticoagulation noted on the discharge summary and $91 \%$ of discharge summaries had a long term anticoagulant plan documented.

Conclusion The electronic prescribing system can help to ensure a clear anticoagulation communication as shown by $91 \%$ of the anticoagulation long term plan being clearly documented; making it a more seamless patient transfer. On the Philips PICU electronic prescribing system there is an anticoagulant section on the discharge summary that has 3 boxes that need to be completed; heparin regimen, indication and anticoagulation long term plan. However, despite these boxes; deviations from the anticoagulant protocol were poorly documented as highlighted by only 33\% having the reason highlighted in the discharge summary, only $50 \%$ of the indications were documented. Despite having prompts for this information on the discharge summary, the medical staffs needs to be aware to complete this information, in order to reduce potential medication errors and risk.

\section{REFERENCE}

1. The National Patient Safety Agency (NPSA). Actions that make anticoagulant therapy safer. NPSA; March 2007.

\section{P045 VALUE FROM YOUR VITAMINS - OPTIMISING VITAMIN SUPPLEMENTATION IN CHILDREN WITH CYSTIC FIBROSIS}

Eleanor Turner. Royal Manchester Children's Hospital

\subsection{6/archdischild-2019-nppc.54}

Aim To optimise fat soluble vitamin supplementation in children with $\mathrm{CF}$ in a tertiary children's hospital and primary care. To compare supplementation of individual vitamins and supplementation with a new multivitamin preparation against national guidelines, cost and patient acceptability. ${ }^{1}$

Methods Comparison of current practice (supplementation of individual vitamins) with a new multi-vitamin preparation, Paravit-CF. Form and dose of each of the fat soluble vitamins was compared against national CF Trust guidelines for nutrition. ${ }^{1}$ Cost of treatment per day was compared. Patient acceptability was explored by comparing medication burden and with a focus group made up of representatives from the CF multi-disciplinary team (MDT) including, consultants, specialist nurses, dieticians and a pharmacist.

Results Current approach to vitamin supplementation is not in line with the CF Trust guidelines for nutrition. Supplementation with Paravit-CF preparations meets recommendations made in the CF Trust guidelines for nutrition for patients of all ages except for infants. Infants will receive vitamin $\mathrm{A}$ at higher doses than recommended. Cost of vitamin supplementation is reduced by approximately 50\% when using Paravit-CF compared with current practice. Medication burden is reduced by more than $50 \%$ when using Paravit-CF compared with 